\title{
THE DYNAMICS OF MARINE CRAFT
}

Maneuvering and Seakeeping 


\title{
ADVANCED SERIES ON OCEAN ENGINEERING
}

\section{Series Editor-in-Chief}

\author{
Philip L- F Liu (Cornell University)
}

\author{
Vol. 5 Numerical Modeling of Ocean Dynamics \\ by Zygmunt Kowalik (Univ. Alaska) and TS Murty (Inst. Ocean Science, BC) \\ $\dot{\mathscr{s}}$ Vol. 6 Kalman Filter Method in the Analysis of Vibrations Due to Water Waves
by Piotr Wilde and Andrzej Kozakiewicz (Inst. Hydroengineering, Polish \\ Academy of Sciences) \\ Vol. 7 Physical Models and Laboratory Techniques in Coastal Engineering \\ by Steven $A$. Hughes (Coastal Engineering Research Center, USA) \\ Vol. 8 Ocean Disposal of Wastewater \\ by lan $R$ Wood (Univ. Canterbury), Robert $G$ Bell (National Institute of Water \\ \& Atmospheric Research, New Zealand) and David $L$ Wilkinson \\ (Univ. New South Wales) \\ Vol. 9 Offshore Structure Modeling \\ by Subrata K. Chakrabarti (Chicago Bridge \& Iron Technical \\ Services Co., USA) \\ Vol. 10 Water Waves Generated by Underwater Explosion \\ by Bernard Le Méhauté and Shen Wang (Univ. Miami) \\ Vol. 11 Ocean Surface Waves; Their Physics and Prediction \\ by Stanislaw $R$ Massel (Australian Inst. of Marine Sci) \\ Vol. 12 Hydrodynamics Around Cylindrical Structures \\ by B Mutlu Sumer and Jørgen Fredsøe (Tech. Univ. of Denmark) \\ Vol. 13 Water Wave Propagation Over Uneven Bottoms \\ Part I - Linear Wave Propagation \\ by Maarten W Dingemans (Delft Hydraulics) \\ Part II - Non-linear Wave Propagation \\ by Maarten Wingemans (Delft Hydraulics) \\ Vol. 14 Coastal Stabilization \\ by Richard Silvester and John R C Hsu (The Univ. of Western Australia) \\ Vol. 15 Random Seas and Design of Maritime Structures (2nd Edition) \\ by Yoshimi Goda (Yokohama National University) \\ Vol. 16 Introduction to Coastal Engineering and Management \\ by $J$ William Kamphuis (Queen's Univ.) \\ Vol. 17 The Mechanics of Scour in the Marine Environment \\ by $B$ Mutlu Sumer and Jørgen Fredsøe (Tech. Univ. of Denmark) \\ Vol. 18 Beach Nourishment: Theory and Practice \\ by Robert G. Dean (Univ. Florida)
}

Vol. 19 Saving America's Beaches: The Causes of and Solutions to Beach Erosion by Scott L. Douglass (Univ. South Alabama)

Vol. 20 The Theory and Practice of Hydrodynamics and Vibration by Subrata K. Chakrabarti (Offshore Structure Analysis, Inc., Illinois, USA) 
Advanced Series on Ocean Engineering - Volume 22

\section{THE DYNAMICS OF MARINE CRAFT}

Maneuvering and Seakeeping

\section{Edward M. Lewandowski}

Computer Sciences Corporation

Washington DC, USA 


\section{Published by}

World Scientific Publishing Co. Pte. Ltd.

5 Toh Tuck Link, Singapore 596224

USA office: Suite 202, 1060 Main Street, River Edge, NJ 07661

UK office: 57 Shelton Street, Covent Garden, London WC2H 9HE

A catalogue record for this book is available from the British Library. సूClearance Center, Inc., 222 Rosewood Drive, Danvers, MA 01923, USA. In this case permission to photocopy is not required from the publisher. ळे in วัISBN 981-02-4755-9

ISBN 981-02-4756-7 (pbk)

Printed in Singapore. 


\section{PREFACE}

This book is intended to serve as an upper-level undergraduate or introductory-level graduate text for students of Naval Architecture or related fields. It is not a book about design of marine vehicles, but rather addresses the question, "How can we predict the dynamic performance of the vehicle, given its physical characteristics?" Thus the material should be of interest to present and future designers, since evaluation of maneuverability/coursekeeping ability and performance in waves is of course an essential (though sometimes neglected) part of the infamous "design spiral" in naval architecture. In addition, the material should also be useful to those interested in simulation of vehicle performance, for training purposes or to conduct engineering studies. The emphasis is on hydrodynamics, since these are the predominant external forces acting on marine vehicles. Knowledge of differential and integral calculus, elementary differential equations, and complex numbers is presumed, as is familiarity with basic fluid mechanics and potential flow theory. The treatment is not intended to be highly mathematical or theoretical; an outline of the theory is given but the emphasis is on exposition of practically useful results. To this end an attempt has been made to present results in the form of equations ("curve fits") rather than plots that do not lend themselves to automatic computation. Several fairly detailed worked examples are included.

Chapter 1 provides a background for the material to follow by introducing coordinate systems and giving the basic form of the equations of motion of a rigid body, with origin at the center of gravity and also at an arbitrary point. (It was my original intention to write a chapter entitled "Introduction" which would precede this and demonstrate the importance and practical usefulness of the material to follow; I ultimately decided that this would be superfluous as this is patently obvious to all). Subsequently, Chapters 2,3 and 5 consider the forces on marine $\checkmark$ vehicles at zero speed (hydrostatics and gravity), at nonzero speed in calm water, fi and in waves (zero and nonzero speed), respectively. Chapter 4 provides the - necessary background in water wave hydrodynamics and the spectral representation $\checkmark$ of ocean waves; those who would like a more thorough treatment should consult IC.C Mei's The Applied Dynamics of Ocean Surface Waves, Volume 1 in this Advanced Series on Ocean Engineering. Chapters $1-5$ constitute a fairly complete coverage of the subject matter for "conventional" marine vehicles (displacement craft and submersibles). Chapter 6 presents supplementary material on the maneuvering and seakeeping performance of "high-speed craft", admittedly biased toward planing monohulls. The formulas presented there, mostly empirical in nature, should be of interest to practitioners but may be "beneath the dignity" of 
theoreticians; however, it will be of use to them for purposes of validation of future theoretical predictions.

One problem that arises in writing a book covering both seakeeping and maneuvering is that traditionally, different coordinate systems have been employed in these two areas: In almost all published works on maneuvering, body-fixed axes $\dot{s}$ are used, with the $\mathrm{x}, \mathrm{y}$ and $\mathrm{z}$ axes pointing forward, to starboard, and downwards, respectively. In seakeeping there is less uniformity, but usually derivations are carried out relative to fixed axes, and the vertical axis is inevitably pointing ¿ुupwards. This is a natural choice since that is the coordinate system used to describe the waves. The maneuvering convention is adopted here as the "primary" g coordinate system; however, most of the material in Chapter 5 is presented relative to "seakeeping axes" with a $\mathrm{z}$-axis pointing upwards. This has necessitated the use Dof several fixed and moving coordinate systems, which unfortunately may cause ×x some confusion. The maneuvering body axes are denoted by $x, y, z$ as usual, and 政 $\xi, \eta, \zeta$ are the corresponding "fixed" axes. In Chapter $4, \xi, \eta, \zeta$ are introduced; these are fixed axes with $\xi, \eta$ lying in the plane of the undisturbed free surface and $\zeta$ Dpointing up. Finally, "seakeeping body axes" $x, y, z$ are applied in Chapter 5; in this E oase $z$ is positive upwards and so $y$ points to port. In problems in which amaneuvering ("steady flow") forces are negligible, you are encouraged to work Exclusively with the seakeeping coordinates. However for simulation of ship . performance we do not in general have the luxury of neglecting steady flow effects; .

In closing I would like to acknowledge the steadfast support of my wife, Donna, and the patience of my daughters Teresa and Janet, throughout the more than five years that it has taken me to finish the book. Completion of this project would not have been possible without their continuous encouragement and understanding. 


\section{CONTENTS}

PREFACE.

CHAPTER 1 DYNAMICS OF RIGID BODIES ................................................... 1

\& 1. Coordinate Systems and Definitions............................................................ 1

\& 2. Angular Displacements and Coordinate Transformations .................................. 3

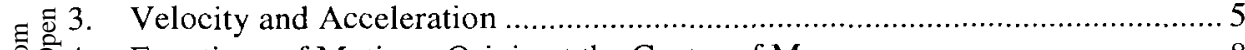

Equations of Motion: Origin at the Center of Mass......................................... 8

5. Equations of Motion: Origin at an Arbitrary Point ........................................ 12

A Third Coordinate System ................................................................... 14

CHAPTER 2 CALM WATER BEHAVIOR OF MARINE VEHICLES AT ZERO SPEED: HYDROSTATICS ........................................... 15

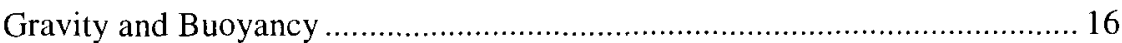

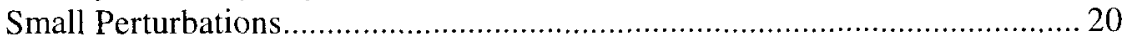

The Restoring Force Coefficient Matrix ..................................................... 24

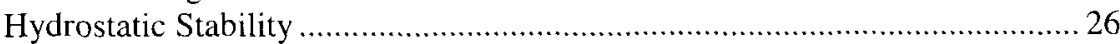

Example: Hydrostatics of a Simple Barge ................................................... 31

CHAPTER 3 CALM WATER BEHAVIOR OF MARINE VEHICLES WITH FORWARD SPEED: MANEUVERING .................................... 35

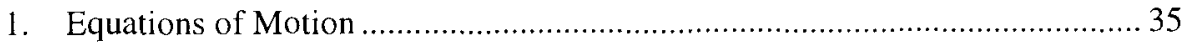

2. Added Mass and Added Moment of Inertia..................................................... 36

2.1 Evaluation of added mass coefficients: Hull ........................................ 40

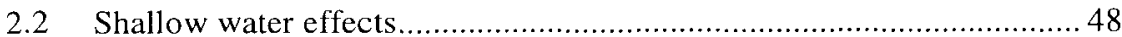

2.3 Evaluation of added mass coefficients: Appendages .......................... 50

2.4 Calculation of added mass: Example ............................................... 51

"Steady" Forces and Moments ......................................................................... 54

Evaluation of Steady Force and Moment Coefficients: Hull ...........................63

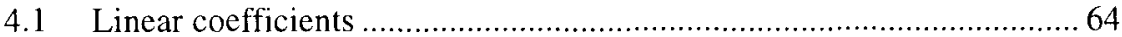

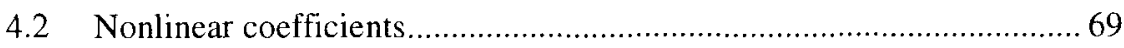

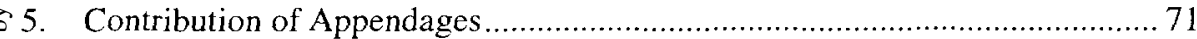

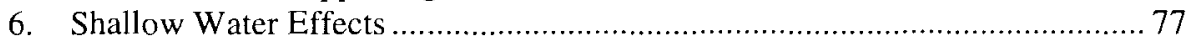

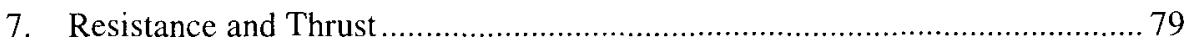

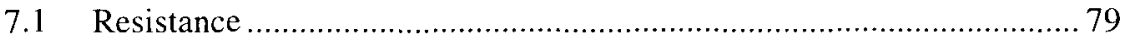

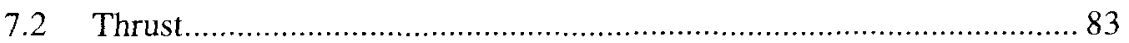




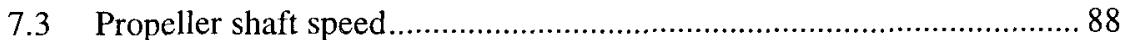

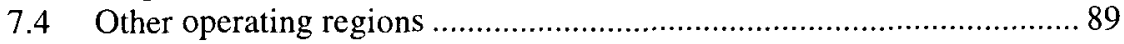

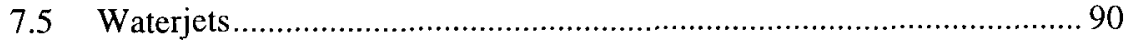

8. Control Forces and Moments...................................................................... 91

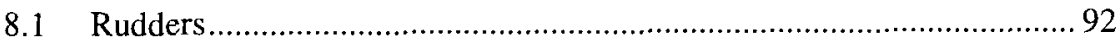

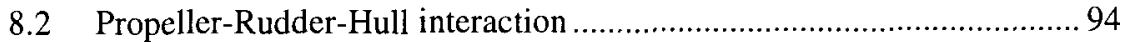

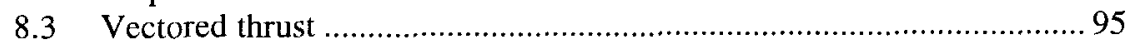

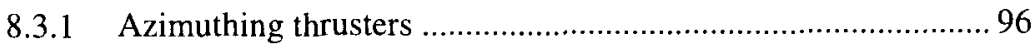

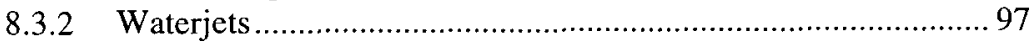

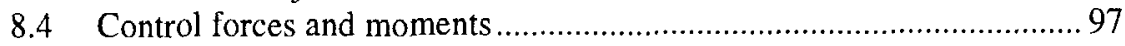

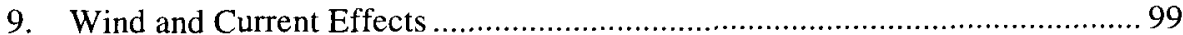

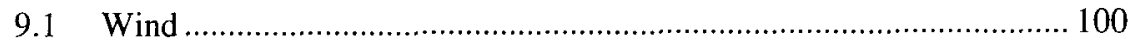

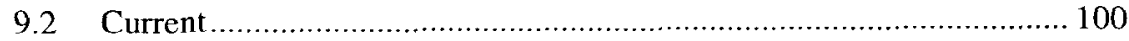

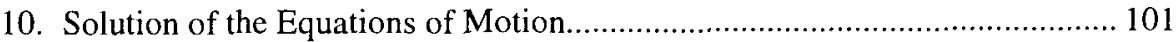

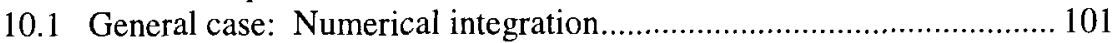

10.2 Solution of the linearized equations; stability ..................................... 108

10.2.1 Horizontal-plane motions .................................................. 110

10.2.2 Example: Controls-fixed stability for horizontal-plane

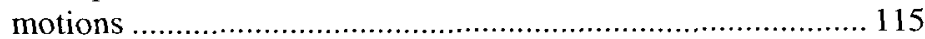

10.2.3 Vertical-plane motions of submersibles.................................. 118

10.2.4 Example: Controls-fixed directional stability for vertical-plane motions........................................................... 121

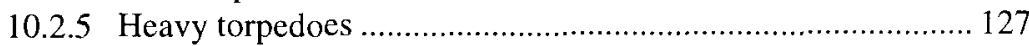

APPENDIX A PREDICTION OF WAKE FRACTION AND THRUST

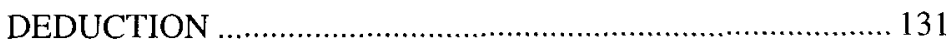

APPENDIX B COEFFICIENTS IN $\mathrm{K}_{\mathrm{T}}$ and $\mathrm{K}_{\mathrm{Q}}$ POLYNOMIALS ................... 135

APPENDIX C ROUTH-HURWITZ STABILITY CRITERION........................ 137

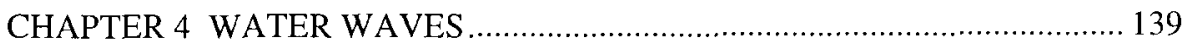

1. A Simple Sinusoidal Wave .......................................................................... 139

1.1 Particle velocities and trajectories; dynamic pressure ........................ 146

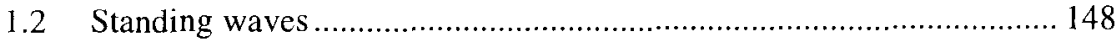

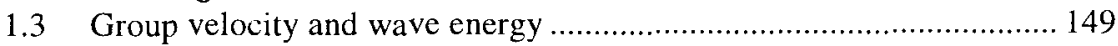

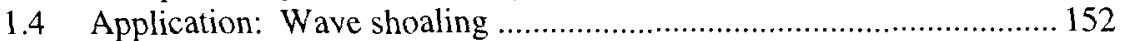

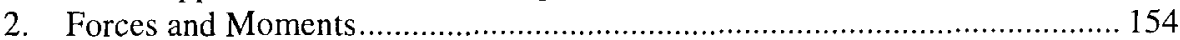

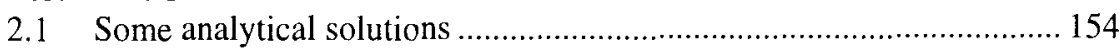

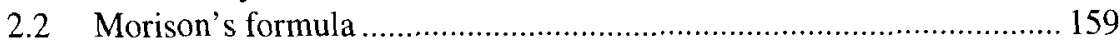

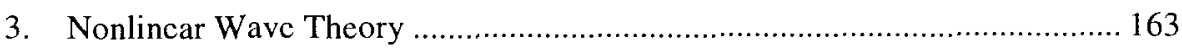

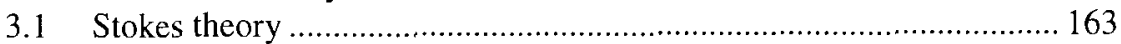

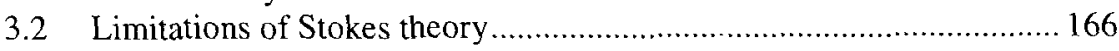

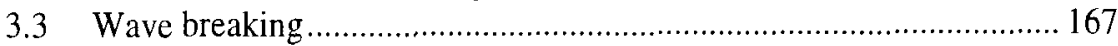


4. Spectral Representation of Ocean Waves .................................................... 168

4.1 Determination of wave spectra ....................................................... 170

4.1.1 Wave spectra from measurements ......................................... 170

4.1.2 Semi-empirical formulations of wave spectra ....................... 172

4.1.3 Statistics of wave heights.................................................... 174

4.2 Representation in the time domain ................................................. 186

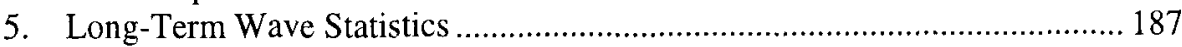

5.1 Maximum waveheight from occurrence data .................................. 187

5.2 Maximum significant waveheight from extreme value distributions

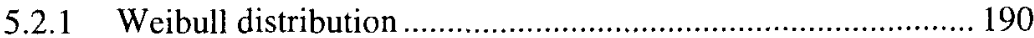

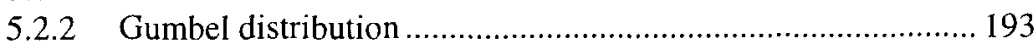

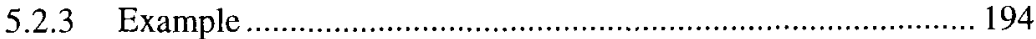

CHAPTER 5 WAVE-INDUCED FORCES ON MARINE CRAFT .................... 199

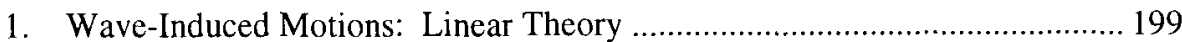

1.1 Hydrodynamic forces: Superposition ................................................2 202

1.2 Equations of motion; simple 1-DOF case .......................................... 204

2. Radiation Forces: Added Mass and Damping ............................................. 209

2.1 General computational procedure, zero speed .................................... 209

2.2 Two-dimensional methods........................................................... 212

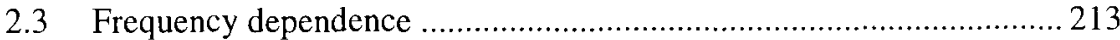

2.4 Added mass and damping forces .................................................... 215

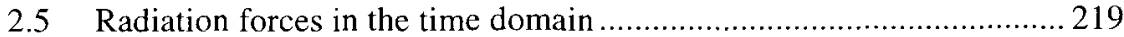

2.6 Effects of forward speed on radiation forces .................................... 222

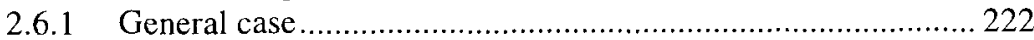

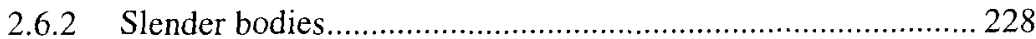

2.7 Transformation to "standard" body axes ............................................ 234

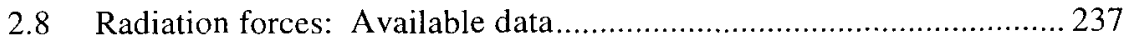

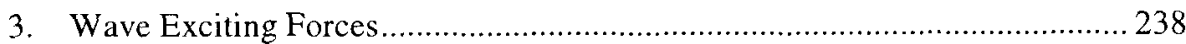

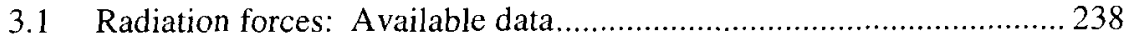

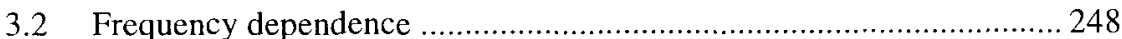

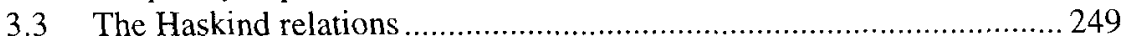

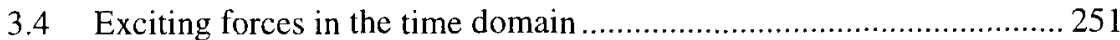

3.5 Effects of forward speed on wave exciting forces .............................. 252

3.5.1 Encounter frequency and encounter spectra .......................... 252

3.5.2 Froude-Krylov force with forward speed.............................. 255

3.5.3 Diffraction force with forward speed ................................... 256

3.6 Transformation to "standard" body axes ............................................... 264

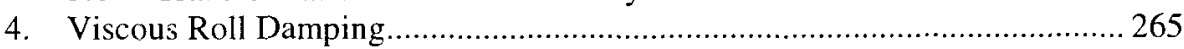

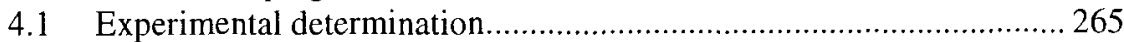

4.1.1 General single degree-of-freedom response ......................... 271 


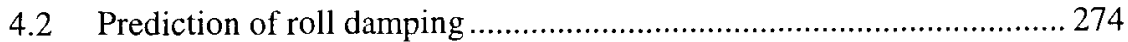

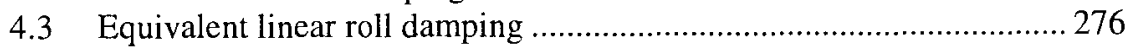

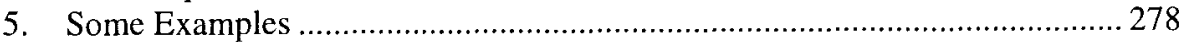

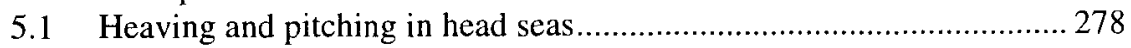

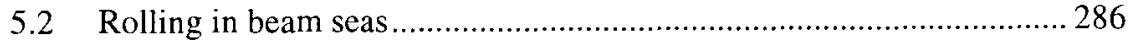

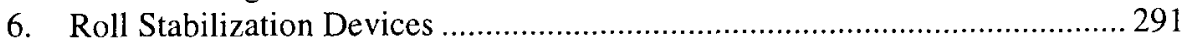

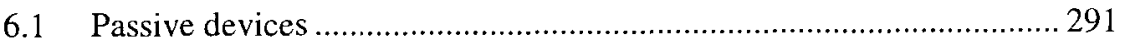

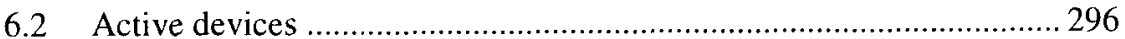

7. Motions in Irregular Waves, Frequency Domain ........................................ 298

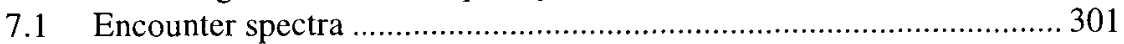

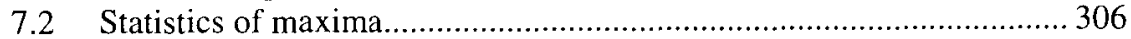

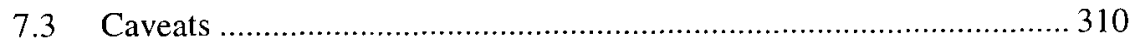

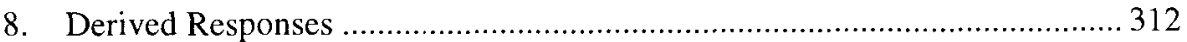

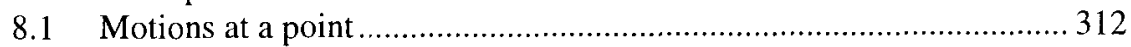

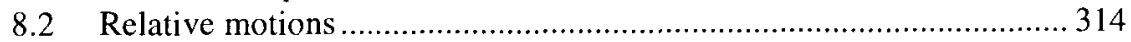

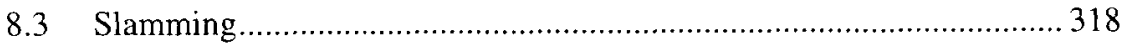

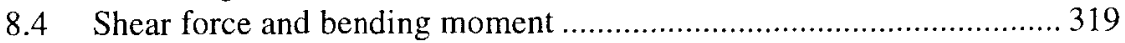

8.5 Motion sickness incidence and motion induced interruptions .............. 320

8.5.1 Motion sickness and fatigue-reduced proficiency.................... 320

8.5.2 Motion induced interruptions.............................................. 324

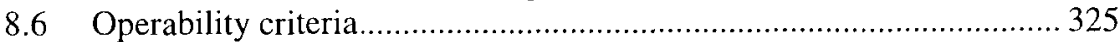

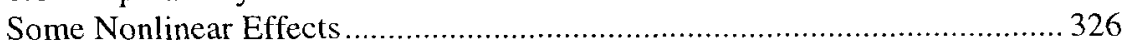

9.1 Evaluation of second order force: Pressure integration ...................... 327

9.2 Evaluation of second order force: Momentum conservation ….......... 332

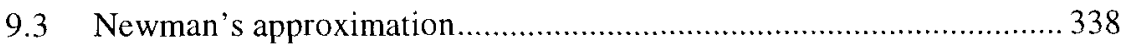

9.4 Effects of forward speed: Wave drift damping and added

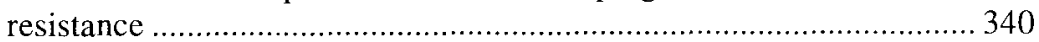

9.4.1 Wave-drift damping ............................................................ 340

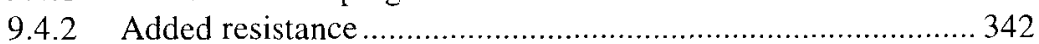

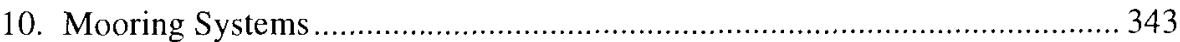

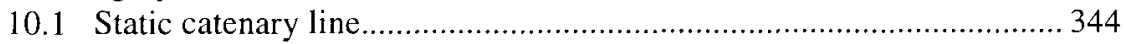

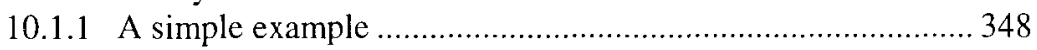

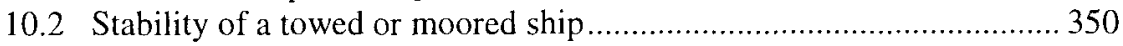

CHAPTER 6 DYNAMICS OF HIGH SPEED CRAFT …..................................... 361

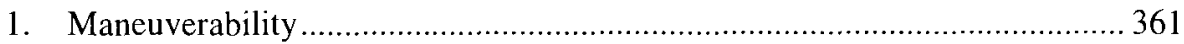

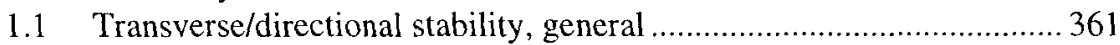

1.2 Transverse/directional stability, planing boats ................................... 366

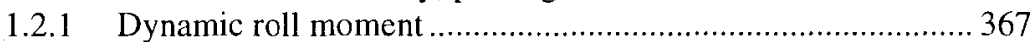

1.2.2 Dynamic stability; effect of appendages ............................... 375

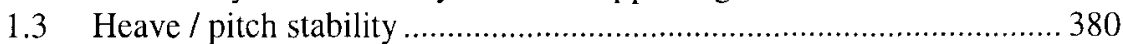

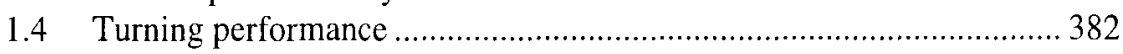




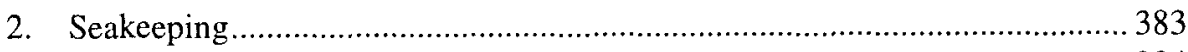

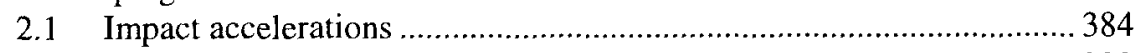

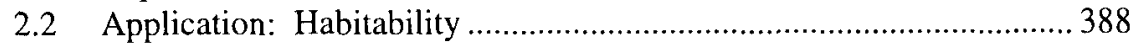

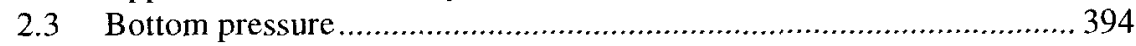

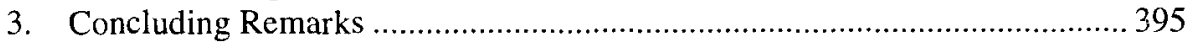

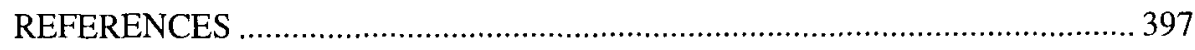

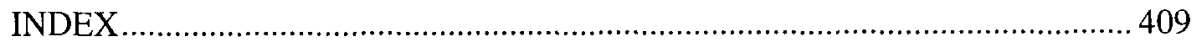

